\title{
SOFT TISSUE MODELLING FROM 3D SCANNED DATA
}

\author{
Jean-Christophe Nebel \\ Department of Computing Science, University of Glasgow, UK
}

Key words: Computer graphics, 3D scanners, FEM, soft tissue modelling.

\begin{abstract}
Human body 3D scanners are becoming a mature technology that generates accurate static photo-realistic 3D models of real human beings. However the data collected allow only the construction of the outer surface of the body. In this paper we describe how from 3D surface data we generate 3D volumetric meshes of soft tissues suitable for the finite element method (FEM). We then deform these meshes using our implementation of the FEM for 3D volumetric meshes. Finally we present some experimental results of the deformation of a human body and detail a methodology to evaluate the distribution of soft tissue layers from a 3D scan of a specific individual.
\end{abstract}

\section{INTRODUCTION}

Human body 3D scanners are becoming a mature technology that generates accurate static photo-realistic 3D models of real human beings. However the data collected allow only the construction of the outer surface of the body. Hence the scans do not have any internal structure and physical properties regarding the skeleton, the skin or the soft tissues of the scanned human. Many works have been done about fitting skeletons in the 3D scans, - segmentation -, in order to allow their animation as articulated figures. However, an other challenging aspect of scan animation is the integration of soft tissue models (skin, muscles, fat...) in these scans for the simulation of the deformation of these soft tissues during motion and interaction within a virtual 3D environment. In order to offer realistic deformations (physically and visually accurate), the 
modelling of soft tissues should be based on the equations of the mechanics using the physical properties of these tissues. Moreover the 3D models representing individuals, the soft tissue properties should be customised according to the person scanned.

In this paper we present how from 3D surface data we generate 3D volumetric meshes of soft tissues suitable for the finite element method (FEM). We then deform these meshes using our implementation of the FEM. Since the 3D scanned data have an accuracy of about $1 \mathrm{~mm}$ and represent specific individuals, our aim is to generate soft tissue deformations customised to these individuals. For that reason, we aim to generate deformations as accurate as possible, whatever the computation time needed. In the future, this work will be used in medical applications.

First we give a review of the techniques used for soft tissue modelling and $3 \mathrm{D}$ scan deformation, then we show how we generate volumetric meshes from 3D scans, we also describe our implementation of the FEM for 3D meshes. Finally we present some experimental results and detail a methodology to get the distribution of fat layers on a specific body.

\section{PREVIOUS WORK}

\subsection{Soft tissue modelling}

Many approaches to soft tissue modelling are based on surface models. Early works restricted themselves to pure geometric deformation [Ko88]. Another approach has been to use models based on implicit surfaces [Ma95], [Mo97] and [Ne98]. These models are composed of a skeletal model upon which parameterised muscles are built up manually and the entire body form is then "skinned," i.e. covered in virtual skin.

However realistic deformations can only be achieved by using physically based models. Surface based models were developed using the finite element method (FEM) for facial animation [Ko98] and surgery simulation [Br96]. They have proved to be very powerful in the context of animation, but they have obvious limitations since they were aimed to show visually convincing deformations.

Two types of volumetric models have been developed depending on the need of interaction with the 3D model. Real time deformations have been demonstrated using models based on the Hooke's law and Lame equation [De99] and [Aubel00] or combining elastic surface and geometric constraints [Tu98]. The most realistic models are based on volumetric mass spring system or FEM simulation. Usually soft tissues are divided in different layers (Skin, fat, muscle...) which have distinct 
physical properties. The first models were based on mass spring system [Te90] and [Le95] and are still used because they have a lower computational cost [Bi99]. The finite element method imposed itself as the most accurate way of simulating soft tissue deformation since nonlinear elasticity and incompressibility can be simulated [La86] and [Ro98].

One of the barriers to using finite element analysis in soft tissue deformation is the generation of the $3 \mathrm{D}$ volumetric mesh on which the simulation will be applied. The mesh generation is a critical feature of the pre-processing stage since the accuracy of the numerical results is strongly related to the quality of the underlying meshes.

\subsection{D scan deformation}

The most effective techniques for generating 3D static photo-realistic models of real human are called scanning techniques. Several methods can be used: laser beams [Tr00] and Cyberware ${ }^{\mathrm{TM}}$, structured light technique [WIO0] or photogrammetry [SiO0] and [Va00]. Their accuracy is usually sufficient for getting very realistic 3D models, whose accuracy is about $1 \mathrm{~mm}$. Moreover colour pictures are mapped on these models what ensures photo realistic appearance. The main difference between the results these full body scanners provide is about the type of data they can capture. Indeed very few of them have short capture time. The scanners, based on laser beams and structured light, have a capture time of about 15 seconds, whereas the ones using photogrammetry, so called 3D imagers, only need few milliseconds. Obviously only the latter type of scanners has the ability to capture subjects which are moving or are in positions which cannot be held for a long time. Hence 3D imagers are unique tools for the investigation of soft tissue modelling.

Whatever the type of scanner used, the data collected allow only the construction of the outer surface of the body, therefor the scans do not have any internal structure and physical properties regarding the skeleton, the skin or the soft tissues of the scanned human.

Many works have been done about fitting skeletons in the 3D scans, segmentation, in order to allow their animation as articulated figures. First that was made by slicing the scans and selecting landmarks manually [Jo95] and [Pa97]. More recently the automatic segmentation of the human body has been investigated too. [Nu97] offered the automatic segmentation of the human body into 6 functional parts ( 2 arms, 2 legs, a torso and a head). This work was refined by focusing on key landmarks of the human body [De98]. Finally [Ju00] worked on segmenting the human body in 16 parts. Although manual interventions may be still needed, 
since the segmentation of extremities is not always accurate enough, these latest results are very encouraging.

Once a skeleton has been fitted inside a 3D model, the vertices of the surface have to be connected to the skeleton. This has been done manually in the game industry for years [La94]. [Su99] has demonstrated a technique for mapping automatically each vertex of a 3D scan to a skeleton placed inside the model to enable seamless animation of the 3D model. Since the purpose of their work is real time animation, the deformation of the model is based only on the relationship between vertices and the skeleton and geometrical constraints. The next step is obviously the integration of realistic soft tissues inside these 3D models.

\section{THE PROPOSED METHOD}

\subsection{Principle}

The soft tissues, we are interested in our research, consist of three elements: the epidermis, the dermis and the subcutis (see Figure 1). The epidermis is the outermost layer that contains the primary protective structure and the dermis is a fibrous layer that supports and strengthens the epidermis. The subcutis is a subcutaneous layer of fat beneath the dermis that supplies nutrients to the other two layers and that cushions and insulates the body; the subcutis is usually connected to muscles.

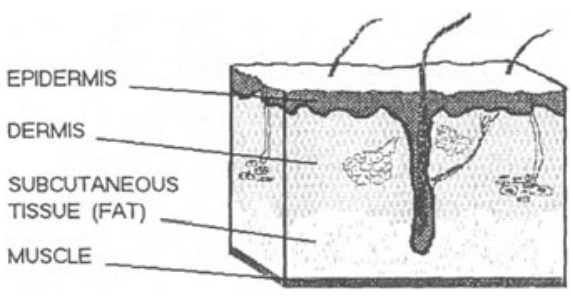

Figure 1. Soft tissue anatomy

Since the anatomy of these soft tissues shows a structure of three layers, volumetric meshes based on this structure are well suited to simulate accurately soft tissues in most parts of the body [Bo00]. However for the fatty parts of the body, e.g. belly and female breasts, another structure should be used: the fatty part should be represented by a volumetric mesh instead of a single layer. We will deal about this case in the next section. 
Scanned data obviously provide the surface of the upper mesh of the structure, we can make the assumption that the thickness of the different layers is constant on a small area. Hence we generate the volumetric mesh by selecting an area of a scan and duplicating this surface mesh to generate the frontiers between the three layers. These meshes, placed in parallel according to the layer thicknesses, are then connected to each other. The meshes generated by 3D scanners are supposed to be composed of triangles, or can be easily converted into triangular meshes, so the connections between two parallel meshes of that kind generate a layer of prism elements. Physical properties are then assigned to each prism element accordingly to the layer it belongs to.

Finally the nodes of the lowest frontier of the fat layer are connected to muscles. Since muscles are very hard - compared to the other soft tissues we assume that they cannot be deformed by realistic and non-destructive external forces. Hence muscles can be deformed only if they move by themselves. Forces are then applied on the exterior of the mesh and deformations are computed using the FEM.

\subsection{Fatty part modelling}

As mentioned previously, the structure used to model the fatty parts of the body (belly, female breast...) should be slightly different from the one presented in the previous section. The layer structure is kept for the epidermis and the dermis, however the subcutaneous fat cannot be modelled by a layer anymore. It should be represented by a volumetric mesh according to the scan data.

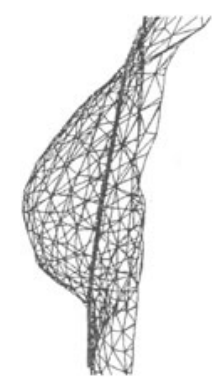

Figure 2. Evaluation of the chest wall

Using the 3D scans, the volume of fat has to be evaluated. In the case of breast simulation we evaluate the position of the chest wall [Mo99] (see Figure 2), we then select the surface defined by the breast and close this surface using a mesh defining the chest wall. The chest wall mesh is 
generated from the points delimitating the breast surface using a $2 \mathrm{D}$ advancing front method. Finally the volumetric mesh modelling the breast fat is generated from the $3 \mathrm{D}$ closed surface representing the surface of the breast using a 3D advancing front method [Ho88], [Ge88], [Ca95] and [Fr96]. The volumetric mesh, which is composed of tetrahedral elements, is then scaled down in order to fit inside the two skin layers generated from the surface mesh.

A more general and detailed presentation of the modelling of the fatty parts of the human body will be offered in a future paper.

\subsection{Finite element model}

The finite element method is a numerical technique that has been applied in many fields. It has become a standard tool in industry and is slowly finding its way into the field of biomechanics.

In this method, the region that is to be analysed is discretised up into sub-regions called elements, these elements are connected at points called nodes. As mentioned previously, our model has to deal with two types of 3D elements: prisms and tetrahedrons. For the time being we consider them as being linear. So their shape functions have standard expressions, $N$, available in most FEM textbooks, i.e. [En99] [Fa99]. The region is represented by functions defined over each element. This generates a number of local functions that are much simpler than those required to represent the entire region. The next step is to analyse the mechanical response for each element. A stiffness matrix, $K$, and a force vector, $F$, are built for each element in the structure, where $U$ is the displacement of each node: $F=K U$

In our model we idealise the soft tissues as elastic, so the standard equation of classical elasticity can be used to express $K$.

We define the following variables:

$\varepsilon$ is the strain of the material

$\sigma$ is the stress of the material

$\mathrm{V}$ is the volume of the element

$D$ is the material property matrix

$B$ is the matrix relating strain to displacement, it depends only on the shape functions, $B=f(N)$.

The governing equations of the model are described as follow: 
Strain-displacement relationship: $\varepsilon=B U$

Strain-stress relationship: $\sigma=D \varepsilon$

Force-stress relationship: $F=\int_{V} B^{T} \sigma d V$

Consequently the expression of $\mathrm{K}$ is: $K=\int_{V} B^{T} D B d V$

For an isotropic 3D element where $E$ is the Young's modulus and $v$ is the Poisson's ratio, the expression of $D$ is:

$$
[D]=\frac{E}{(1+v)(1-2 v)}\left[\begin{array}{cccccc}
1-v & v & v & 0 & 0 & 0 \\
v & 1-v & v & 0 & 0 & 0 \\
v & v & 1-v & 0 & 0 & 0 \\
0 & 0 & 0 & \frac{1-2 v}{2} & 0 & 0 \\
0 & 0 & 0 & 0 & \frac{1-2 v}{2} & 0 \\
0 & 0 & 0 & 0 & 0 & \frac{1-2 v}{2}
\end{array}\right]
$$

Once all the element stiffness matrices and force vectors have been obtained they are combined into a structure matrix equation. This equation relates nodal displacements for the entire structure to nodal loads. After applying boundary conditions the structure matrix equation can be solved to obtain unknown nodal displacements. Intra-element displacements can be interpolated from nodal values using the functions that were defined over each element.

\section{RESULTS AND DISCUSSION}

\subsection{Deformation of an upper arm}

Since our work is aimed at medical applications, where simulations are limited to specific areas of the body, we demonstrate our method through the deformation of an upper arm on which a force is applied. 


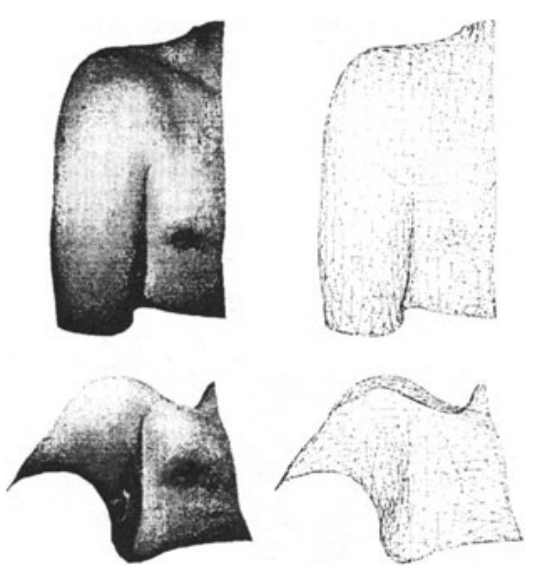

Figure 3. 3D model of a shoulder and upper arm

At first the geometry of the 3D volumetric mesh has to be generated. The 3D surface and the appearance of the right shoulder and upper arm of the author are captured using the C3D imager [Si00] (see Figure 3). A piece of the upper arm is cut out of the generated 3D model (see Figure 4), this mesh is used as a base for the construction of the 3D volumetric mesh.
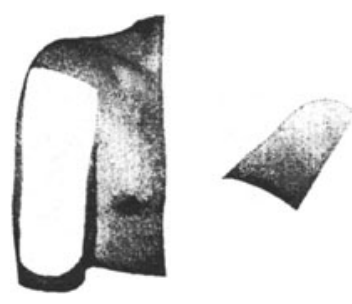

Figure 4. Collection of the 3D surface

We duplicate the surface mesh to generate the three layer volumetric mesh (see Figure 5). The thicknesses of the epidermis, dermis and subcutaneous fat are set respectively, using average values, at $0.2 \mathrm{~mm}$, 2mm [Ru66] [Ho74] and 8.9mm [Fr81]. 


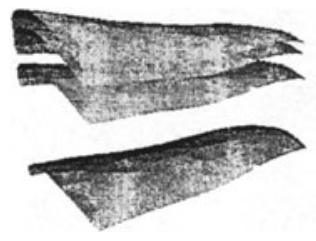

Figure 5. Volumetric mesh (not at scale)

Then we set physical properties to these materials. Since they are incompressible [La87], their Poisson's ratios are set at 0.50 . Finally their Young's moduli are set respectively at $90.10^{9} \mathrm{~N} . \mathrm{m}^{2}, 45.10^{9} \mathrm{~N} . \mathrm{m}^{2}$ and $30.10^{9} \mathrm{~N} \cdot \mathrm{m}^{2}$.

In order to apply the FEM on that volumetric mesh, boundary conditions have to be defined. The lowest layer (fat layer) is fixed to muscles (deltoid, biceps and triceps) and an external force is applied on the mesh $(0.15 \mathrm{~N})$. Then the deformation is computed (see Figure 6).
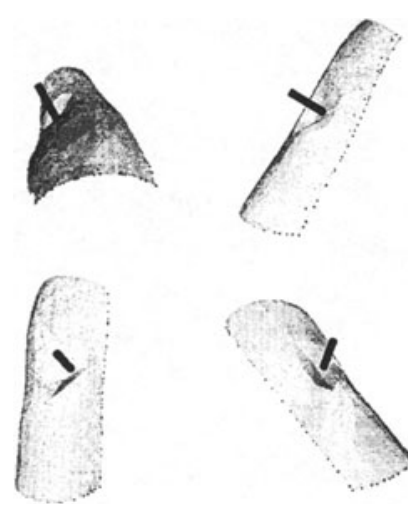

Figure 6. Deformed mesh seen from different view points

Finally the deformed mesh is put back inside the initial 3D model. In Figure 7, we show the initial and final meshes of the shoulder and upper arm without texture and with texture and different illuminations. 


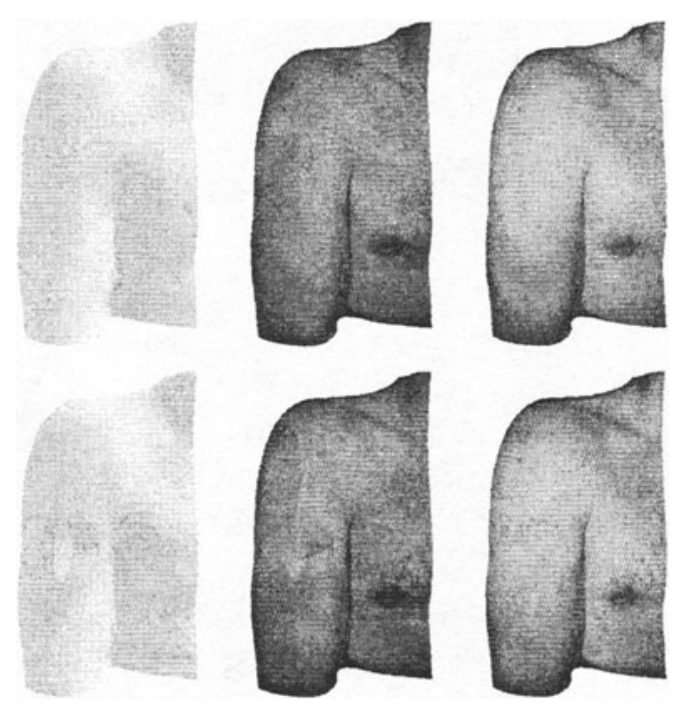

Figure 7. Deformed 3D model

\subsection{Discussion}

These results demonstrate our method for generating and deforming realistic 3D volumetric mesh. This method is automatic once the area of interest and forces have been specified. Moreover, visually, the deformation looks quite realistic.

Since the data used for the fat layer thickness is an average value, it does not make much sense to compare precisely the simulated deformation with the deformation that similar conditions would generate on the real human. That limitation occurs because of the lack of data to evaluate the distribution of fat layers on a specific individual.

The accuracy of a simulation using FEM depends on the accuracy of the geometry of the mesh representing the soft tissues and the knowledge of the physical properties of these tissues. The physical properties of the human skin and fat are very well known [Da82], [La87]. For example, regional differences of skin thickness have been extensively studied [Ru66], [Ho74]: the two first layers of human skin (dermis and epidermis) varies in thickness from $0.5 \mathrm{~mm}$ on eyelids to more than $5 \mathrm{~mm}$ on the middle of the upper back.

However the thickness of fat layers varies a lot depending on the localisation and especially on the individual (few $\mathrm{mm}$ to few $\mathrm{cm}$ ). Its evaluation relies on measurements on the individual. On one hand, the most efficient methods are either invasive or require expensive material (bioelectrical impedance analysis, X-rays, CT and MRI). On the other 
hand, skin fold measurement is a simple and cheap method for estimation of the depth of the fat layer [Hi79]. The drawback of this method is that it is a manual and tedious task, since hundreds of measurements are needed to get a realistic evaluation of the distribution of fat layers on a specific body.

We can investigate another method that would be more suitable for our application: anthropometric measurements (girths and lengths), is a quick method used to estimate body composition. By measuring 11 sites on a body, calculations of percent body fat [Ho92] and weight [Be74] can be evaluated. Moreover three other indices, the endomorphy (roundness), the mesomorphy (muscularity) and the ectomorphy (lankyness), can be calculated to get a better picture of the physical shape of an individual [Ca91].

Using a set of models whose fat distribution and key indices are known, we should be able to interpolate the fat distribution for any new individual. Since we are currently working on automatic anthropometric measurements on full body scans using segmentation and landmark recognition techniques [De98] and [Ju00] this process of fat distribution evaluation could become fully automatic.

\section{CONCLUSION AND FUTURE WORK}

We presented a full process allowing realistic deformation of 3D scanned data based on the FEM applied on volumetric meshes. The generation of the volumetric mesh is automatic once the area of interest has been specified. Moreover that technique can be applied on any scanned data since there are no constraints regarding the initial mesh.

Results were demonstrated, the present limitations were discussed and details were given about a way of getting the distribution of fat layers on a specific body.

We are currently working on this specific issue. Once this investigation completed we will be able to evaluate the limitations generated by our linear model for soft tissue deformation.

\section{REFERENCES}

[Au00] A. Aubel, D. Thalmann, Realistic Deformation of Human Body Shapes, Proc. Computer Animation and Simulation 2000, Interlaken, 2000

[Be74] A. R. Behnke and J. H. Wilmore, Evaluation and regulation of body build and composition, Prentice-Hall, USA, 1974 
[Bi99] D. Bielser, V. A. Maiwald and M. H. Gross, Interactive cuts through 3-dimensional soft tissue, Computer Graphics Forum, 18(3), 1999

[Bo00] L. Boissieux, G. Kiss, N. Magnenat Thalmann and P. Kalra, Simulation of skin aging and wrinkles with cosmetics insight, Computer animation and simulation'00, 2000

[Br96] M. Bro-Nielsen and S. Cotin. Real-time volumetric deformable models for surgery simulation using finite elements and condensation, Computer Graphics Forum, 15(3), pp 57-66, 1996

[Ca91] JEL Carter \& BH Heath, Somatotyping: Developments and Applications. Cambridge University Press, New York, 1991.

[Ca95] M. J. Castro Díaz and F. Hect, Anisotropic Surface Mesh Generation, INRIA Research, Report, No 2672, 1995.

[Da82] C. H. Daly, Biomechanical properties of dermis, the journal of investigative dermatology, Vol. 79, pp 17-20, 1982

[De98] L. Dekker, S Khan, E. West, B. Buxton and P. Treleaven. Models for understanding the 3D human body form. Proc. IEEE workshop on model-based 3D image analysis, pp 65-74, Bombay, India, 1998

[De99] G. Debunne, M. Desbrun, A. Barr and M.-P. Cani, Interactive multiresolution animation of deformable models, Computer animation and simulation'99, 1999

[En99] K. M. Entwistle, Basic principles of the finite element method, London : IOM Communications, 1999

[Fa99] M. J. Fagan, Finite element analysis, Longman 1999

[Fr81] J. Frank, A. M. Klidjian and S. J. Karran, The radiological assessment of arm muscle and fat stores in normal and malnourished patients, Clinical radiology, 32, pp 467-470, 1981

[Fr96] P. J. Frey and H. Borouchaki, Delaunay tetrahedralization using an advancing-front approach, in Proceedings of the Fifth International Meshing Roundtable, Pittsburgh, PA, 1996.

[Ga54] S. M. Garn, Fat paterning and fat intercorrelations, Human biology, 26 ,pp 59-69, 1954

[Ge88] P. L. George and H. Borouchaki, Delaunay Triangulation and Meshing Application to Finite Elements, Editions HERMES, Paris, 1998.

[J095] R. R. M. Jones, P. Li, K. Brook-Wavell and G. M. West, Format of human body modelling from 3D body scanning, International journal of clothing science, 7(1), pp 7-16, 1995

[Ju00] X. Ju, N. Werghi and P. Siebert, Automatic Segmentation of 3D Human Body Scans, IASTED International Conference on Computer Graphics and Imaging 2000 (CGIM 2000), 19-23 Nov. 2000, Las Vegas, USA.

[Ko88] K. Komatsu, Human skin model capable of natural shape variation, The visual computer, Vol. 3, pp 265-271, 1988

[Ko98] R. M. Koch, M. H. Gross and A. A. Bosshard. "Emotion editing using finite element models." In Proc. Eurographics'98, 1998.

[Hi79] J. H. Himes, A. F. Roche and R. M. Siervogel, Compressibility of skinfolds and the measurement of subcutaneous fatness, Am J Clinical Nutrition, Vol. 32, pp 1734-1740, 1979

[Hi80] J. H. Himes, A. F. Roche and P. Webb, Fat areas as estimates of total body fat, The American journal of clinical nutrition, vol. 33, pp 2093-2100, 1980

[Ho74] K. A. Holbrook and G. F. Odland, Regional differences in the thickness (cell layers) of the human stratum corneum: an ultrastructural analysis.

[Ho88] K. Ho-Le, Finite element mesh generation methods: a review and classification, Computer Aided Design, Vol 20(1), 27-38, 1988. 
[Ho92] J. A. Hodgdon, Body composition in the military services: standards and methods, in Body composition and physical performance 1992, pp 55-70, National Academy Press, 1992

[La86] W. F. Larrabee, A finite element model of skin deformation, Laryngoscope, 96, pp 399-419, 1986

[La87] Y. Lanir, Skin mechanics, Chapter 11, in Handbook of Bioengineering, McGraw-Hill, USA, 1987

[La94] J. Lander, Skin them bones: game programming for the web generation, Game developer, May, 1994

[Le95] Y. Lee, D. Terzopoulos and K. Waters, Realistic modeling for facial animation Computer graphics, Vol. 29, pp 55-62, 1995

[Ma95] Magnenat Thalmann N, Thalmann D., Proc.IEEE, Switzerland, 1995. " Digital Actors for Interactive Television ".

[Mo97] L.Moccozet, N.Magnenat-Thalmann, "Multilevel Deformation Model Applied to Hand Simulation for Virtual Actors", VSMM97, Geneva, Switzerland, 1997.

[Mo99] R. A. Moffett, A Prototype 3D Breast Surgery Planning and Assessment Tool, MSc IT, Department of Computing Science, University of Glasgow, Glasgow, October 1999.

[Ne98] L. P. Nedel and D. Thalmann. Modeling and deformation of the human body using an anatomically based approach.

[Nu97].J. H. Nurre, Locating landmarks on human body scan data. International conference of recent advances 3D digital imaging and modelling, pp 289-295, 1997, IEEE NJ, USA

[Pa97] R. P. Pargas, N. J. Staples and J. S. Davis, Automatic measurement extraction for apparel from a 3D body scan, Optics and Lasers in Engineering, 28(2), pp 157-172, 1997

[Ro98] S. H. M. Roth, M. H. Gross, S. Turello and F. R. Carls, A Bernstein-Bezier based approach to soft tissue simulation, Computer Graphics Forum, 17(3), 1998

[Ru66] R. F. Rushmer, K. J. K. Buettner, J. M. Short and G. F. Odland, The skin, Science, 154(3747), pp 343-348, 1966

[Si00] J. Paul Siebert and Stephen J. Marshall, Human body 3D imaging by speckle texture projection photogrammetry, Sensor Review, 20 (3), pp 218-226, 2000.

[Su99] W. Sun, A. Hilton, R. Smith and J. Illingworth, Building layered animation models from captured data, Computer Animation and Simulation 99, Springer Computer Science, pp 145-154, 1999

[Te90] D. Terzopoulos, Physically based facial modelling, analysis and animation, The journal of visualization and computer animation, Vol. 1, pp 73-90, 1990.

[Tr00] R. Trieb, 3D-Body Scanning for mass customized products - Solutions and Applications, International Conference of Numerisation 3D - Scanning 2000, 24-25 May 2000, Paris, France.

[Tu98] R. Turner and E. Gobbetti. Interactive construction and animation of layered elastically deformable characters, Computer Graphics Forum, 17(2), pp 135-152, June 1998.

[Va00] G. Vareille, Full body 3D digitizer, International Conference of Numerisation 3D Scanning 2000, 24-25 May 2000, Paris, France.

[Wi00] S. Winsborough, An insight into the design, manufacture and practical use of a 3DBody Scanning system, International Conference of Numerisation 3D - Scanning 2000, 24-25 May 2000, Paris, France. 